\title{
Programa Intergeracional de Estimulação Cognitiva: Benefícios Relatados por Idosos e Monitores Participantes
}

\author{
Rodrigo de Rosso Krug ${ }^{1, *}$ (D), Lariane Mortean $\mathrm{Ono}^{2}$ (D), Thamara Hübler Figueiró ${ }^{3}$ (D), \\ André Junqueira Xavier ${ }^{4}$ (D), \& Eleonora d'Orsi ${ }^{3}$ (D) \\ ${ }^{1}$ Universidade de Cruz Alta, Cruz Alta, RS, Brasil \\ ${ }^{2}$ Universidade Federal do Paraná, Curitiba, PR, Brasil \\ ${ }^{3}$ Universidade Federal de Santa Catarina, Florianópolis, SC, Brasil \\ ${ }^{4}$ Universidade do Sul de Santa Catarina, Palhoça, SC, Brasil
}

\begin{abstract}
RESUMO - Investigaram-se os benefícios percebidos pelos idosos e monitores participantes do programa intergeracional de manutenção, estimulação e/ou reabilitação cognitiva, mediado pelo uso de computadores e prática de atividades físicas "Oficina da Lembrança". Realizou-se uma pesquisa qualitativa descritiva, com dez idosos e seis monitores participantes da Oficina da Lembrança. Aplicou-se entrevista semiestruturada, interpretada pela análise de conteúdo. Os idosos relataram: aprendizagem do uso de computadores, melhora da memória, sociabilização, ter atividade de lazer, emagrecimento, melhora da qualidade de vida, saúde e sociabilização. Monitores: contato com idosos, melhora da relação com pessoas/pacientes, preparação para situações diversas e conhecimentos sobre idosos. Conclui-se que a Oficina da Lembrança pode proporcionar vários benefícios para seus praticantes e para seus monitores, principalmente a intergeracionalidade.
\end{abstract}

PALAVRAS-CHAVE: idosos, intergeracionalidade, estimulação cognitiva, atividade motora, bem-estar percebido, qualidade de vida

\section{Intergenerational Cognitive Stimulation Program: Benefits Reported by Elderly and Monitors Participating}

\begin{abstract}
We investigated the benefits perceived by older people and monitors participants of the intergenerational program intergenerational of maintenance, stimulation/rehabilitation cognitive, mediated by the use of computers and physical activity "Oficina da Lembrança". We performed a descriptive qualitative research with ten elderly and six monitors participants of Oficina da Lembrança. We applied semi-structured interviews, which were interpreted by content analysis. The elderly related: learning the use of computers, improved memory, socialization, having leisure activity, weight loss, improved quality of life, health and socialization. Monitors: contact with elderly, improvement of the relationship with people and patients, preparation for different situations and acquiring more knowledge about the elderly. In conclusion, Oficina da Lembrança can provide several benefits to its practitioners and monitors, especially the intergenerationality.

KEYWORDS: elderly, intergenerationality, cognitive stimulation, motor activity, perceived well-being, quality of life
\end{abstract}

Com aumento da expectativa de vida observado, há maior exposição a fatores de risco e, consequente, suscetibilidade ao desenvolvimento de agravos crônicos à saúde dos idosos, especialmente queixas de memória, problemas cognitivos e demência (American College of Sports Medicine, 2009; Fonseca et al., 2015; SinghManoux et al., 2012; World Health Organization, 2012 ). A demência se caracterizada pela perda global e progressiva das funções cognitivas, com comprometimento significativo da memória, aprendizagem, linguagem, orientação e funções executivas e é considerada a principal causa de dependência e incapacidade entre os idosos, além de contribuir para o desenvolvimento de morbidades e comprometimento da saúde, elevando os custos com assistência médica e social

*E-mail: rodrigo_krug@hotmail.com

- Submetido: 18/04/2016; Revisado: 28/03/2018; Aceito: 02/05/2018. 
(World Health Organization, 2012). Em 2011, o número estimado de pessoas vivendo com demência foi de 35,6 milhões. Espera-se que esse número chegue a 65,7 milhões em 2030. No Brasil, projeções apontam que mais de 7,9\% da população com 65 anos ou mais apresentará demência em 2020 (Burlá, Camarano, Kanso, Fernandes, \& Nunes, 2013).

Diversos fatores relacionados ao estilo de vida estão sendo citados como possíveis fatores de risco para a demência, sendo que $35 \%$ da demência pode ser atribuída a uma combinação desses fatores. Dentre eles, podemos citar o nível de escolaridade e socioeconômico, perda auditiva, hipertensão, obesidade, tabagismo, depressão, inatividade física, diabetes e isolamento social (Livingston et al., 2017).

Parece ainda que a exposição cumulativa a fatores que aumentam a reserva cognitiva, como nível socioeconômico, escolaridade e complexidade no trabalho ao longo da vida reduz o risco de demência no final da vida, mesmo entre indivíduos com predisposição genética à demência (Wang, MacDonald, Dekhtyar, \& Fratiglioni, 2017).

Assim, torna-se necessário um maior cuidado em relação à realidade da saúde mental de pessoas idosas, sendo estimuladas práticas de intervenção (Zanello, Silva, \& Henderson, 2015). Nesse sentido, a manutenção, estimulação e/ou reabilitação da função cognitiva são importantes para a saúde geral das pessoas e podem promover melhora da qualidade de vida e independência (Ngandu et al., 2015; Singh-Manoux et al., 2012), pois auxiliam na redução das limitações físicas, mentais e socioeconômicas advindas do aumento da idade, aumentando a cidadania, saúde, educação, trabalho, lazer, sociabilização, aumentam a comunicação com familiares e amigos, favorecem novas amizades e fortalecem as relações intergeracionais (Ala-Mutka, Malanowski, \& Punie, 2008).

Atividades cognitivamente estimulantes, prática de atividades físicas, controle de doenças, bem como ter uma dieta saudável e não fazer uso de tabaco ou abusar de álcool, podem impedir um em cada três casos de demência (Norton, Matthews, Barnes, Yaffe, \& Brayne, 2014). Assim, o uso de computadores e a prática de atividades físicas vêm se mostrando boas alternativas terapêuticas para manutenção, estimulação e/ou reabilitação da função cognitiva (Barnes et al., 2013; Ngandu et al., 2015).

Muitos estudos já mostram que o uso de internet pode ter efeito de retardar o declínio cognitivo ou até mesmo promover a função cognitiva (Cotelli, Manenti, Zanetti, \& Miniussi, 2012; Lautenschlager \& Cox, 2013; Tardif \& Simard, 2011; Woods, Aguirre, Spector, \& Orrell, 2012). O uso de internet, além de fortalecer os laços sociais, tem o potencial de auxiliar na manutenção da capacidade cognitiva, melhorando as funções executivas e memória episódica (Klusmann et al., 2010). Estudo de revisão sistemática de Diamond e Ling (2015), que analisou 84 pesquisas que tinham objetivo de avaliar diferentes tipos de programas de reabilitação cognitiva, evidenciou que o uso de internet melhora a criatividade, a flexibilidade cognitiva, atenção, execução de tarefas e outras habilidades cognitivas.

Em um estudo populacional longitudinal, identificouse estabilização e/ou retardo do declínio cognitivo, resultante do uso cotidiano de internet. Isso ocorreu tanto em indivíduos de meia idade, como em idosos e, especialmente, em pessoas com pior função cognitiva (Xavier et al., 2014).

A prática de atividades físicas também pode ser considerada uma boa forma de reabilitação da função cognitiva, pois melhora a atenção, memória, função executiva, aumenta o transporte de oxigênio para o cérebro, a síntese e a degradação de neurotransmissores, melhora o tempo de resposta e reprime o declínio da função cardiovascular associado ao declínio cognitivo (American College of Sports Medicine, 2009; Barnes et al., 2013).

Estudo de metanálise de Blondell, Hammersley-Mather e Veerman (2014) analisou vinte e uma pesquisas sobre atividade física e declínio cognitivo e vinte e seis pesquisas sobre atividade física e demência, todos publicados de 2007 a dezembro de 2013, e concluiu que as pessoas que tinham maior nível de atividade física apresentavam menor risco de declínio cognitivo $(\mathrm{RR}=0,65$; IC95\% $=0,55-0,76)$ e demência $(\mathrm{RR}=0,86$; IC95\% $=0,76-0,97)$, quando comparados aos com menores níveis.

Sob a perspectiva acadêmica, programas de extensão com foco na população idosa fornecem aos discentes a oportunidade de implementar atividades que promovam mudanças sociais importantes na vida dessa população, a partir do conhecimento teórico-prático adquirido ao longo da graduação, ao mesmo tempo, essa prática surge como meio de interação entre alunos, professores e comunidade, favorecendo o desenvolvimento pessoal e profissional dos estudantes (Fórum de Pró-reitores de Extensão das Universidades Públicas Brasileiras, 2012).

Sendo assim, analisar os benefícios percebidos pelos participantes de programas intergeracionais de manutenção, estimulação e/ou reabilitação cognitiva permite avaliar a satisfação e autopercepção de bem estar e ganhos subjetivos de um novo método de trabalho cognitivo, que alia o uso de computadores e atividades físicas e auxilia no melhor planejamento para esse tipo de intervenção, além de proporcionar maior entendimento do impacto dessas mudanças na vida dos idosos e de jovens em processo de formação profissional. Este estudo teve como objetivo analisar os benefícios percebidos pelos idosos participantes de um programa intergeracional de manutenção, estimulação e/ou reabilitação cognitiva e os benefícios percebidos pelos monitores do programa para sua formação profissional. 


\section{MATERIAIS E MÉTODOS}

\section{Tipo de estudo}

Este estudo qualitativo descritivo faz parte do projeto de extensão "Oficinas de inclusão digital, estimulação e reabilitação cognitiva de idosos: Oficina da Lembrança", da Universidade Federal de Santa Catarina (UFSC) e foi conduzido em Florianópolis/SC, entre 2 de setembro e 12 de dezembro de 2014.

\section{Sujeitos do Estudo}

Os participantes deste estudo foram idosos com 60 anos ou mais, oriundos do inquérito denominado EpiFloripa Idosos, uma coorte prospectiva, observacional e de base populacional, realizado em Florianópolis/SC, em 2009/2010 (primeira onda) e 2013/2014 (segunda onda). A amostra foi inicialmente de 1702 idosos, residentes na zona urbana da cidade. Na segunda onda, participaram do estudo 1197 idosos. Idosos que responderam positivamente questões sobre queixas de memória e interesse em participar de um programa de estimulação cognitiva foram sorteados aleatoriamente e convidados a participar da Oficina da Lembrança.

Dentre os sorteados e contatados, 15 aceitaram participar e desses 10 obtiveram uma frequência maior ou igual a $50 \%$ e participaram do projeto até o último dia (perda de 33,33\%). Foi considerado critério de inclusão ser participante do Estudo EpiFloripa Idoso e de exclusão a frequência nas Oficinas inferior a $50 \%$.

Em relação aos monitores, foram selecionados oito monitores, todos alunos do curso de Medicina da UFSC, quatro deles contemplados com bolsas de extensão (Programa de Bolsas PROEX/UFSC) e quatro voluntários. Desses, dois foram excluídos por não comparecerem regularmente ao projeto e não cumprimento de atividades propostas.

\section{A Oficina da Lembrança}

O programa de manutenção, estimulação e/ou reabilitação cognitiva, denominado "Oficina da Lembrança" foi idealizado pelo geriatra André Junqueira Xavier, no ano de 2000, através de sua dissertação de mestrado do Programa de Pós-Graduação em Ciências da Computação da Universidade Federal de Santa Catarina (UFSC), com o objetivo de analisar o processo de interação de um grupo de idosos por meio da Internet (Xavier, 2002). Com o passar dos anos, a Oficina da Lembrança foi implementada em outras instituições, como na Universidade Federal de São Paulo (UNIFESP), Universidade do Sul de Santa Catarina (UNISUL) de Tubarão/SC e Palhoça e retomada na UFSC. Inicialmente, o programa envolvia o uso de computadores, alongamentos e discussão em grupos intergeracionais. A partir de 2013, o programa inclui em seu roteiro a prática de atividades físicas, com o objetivo de unir a melhor evidência disponível para o trabalho.

A Oficina da Lembrança era composta pela sequência de 20 a 30 minutos de atividades no computador, seguidos por 20 a 25 minutos de atividades físicas e, posteriormente, por mais 20 a 30 minutos de uso do computador. Ao final, encerra-se com relatos individuais orais e compartilhamento das experiências vividas no encontro (10 a 15 minutos).

Neste estudo, foram analisados apenas os idosos participantes da Oficina da Lembrança da UFSC no ano de 2014/2, primeira turma do projeto na UFSC após sua retomada. Os encontros ocorreram no laboratório de informática do Centro de Ciências da Saúde da UFSC. Ao todo, foram realizados 22 encontros (aproximadamente duas vezes/semana), ministrados por monitores previamente treinados, supervisionados por dois professores do Programa de Pós-Graduação em Saúde Coletiva da UFSC e um profissional de Educação Física.

O roteiro baseou-se no uso do mouse, browser, navegação, email, ferramentas sociais e de desenho livre, apresentador de fotos e jogos. As atividades desenvolvidas em cada oficina estão descritas no Quadro 1.

A atividade física foi ministrada por um profissional de Educação física e era composta por exercícios aeróbicos (caminhada, dança, circuitos, etc), brincadeiras com e sem material de apoio (cones, bolas e alteres de $1 \mathrm{Kg}$ ), exercícios localizados com pesos e alongamentos globais. Maiores informações sobre a Oficina da Lembrança podem ser lidas em estudo previamente publicado (Krug et al., 2015).

\section{Instrumentos e Coleta de Dados}

A coleta de informações com os idosos e monitores foi realizada diariamente ao final de cada encontro, quando os mesmos faziam relatos individuais orais sobre as experiências vividas no encontro. Essas informações foram anotadas em um diário de campo por um dos monitores que era previamente escolhido.

Ao final dos 22 encontros, foi aplicada, em uma sala separada, de forma individual, a entrevista semiestruturada para identificar os benefícios percebidos pelos idosos e monitores. Nos idosos, o instrumento foi aplicado pelos monitores, que foram previamente capacitados pelos supervisores do projeto. O roteiro da entrevista foi o seguinte: 1- Qual(is) o(s) benefício(s) que o senhor(a) percebeu ao participar da Oficina da Lembrança?; 2- Qual(is) o(s) benefício(s) que o senhor(a) percebeu a fazer uso do computador?; 3- Qual(is) o(s) benefício(s) que o senhor(a) percebeu ao praticar atividades físicas?

Nos monitores, o instrumento foi aplicado pelos supervisores do projeto. O roteiro foi o seguinte: 1- Qual(is) $\mathrm{o}(\mathrm{s})$ benefício(s) de ter sido monitor do projeto em sua 
Descrição das atividades realizadas na Oficina da Lembrança, Florianópolis, Santa Catarina, Brasil, 2014

\begin{tabular}{ll}
\hline Oficina & \multicolumn{1}{c}{ Atividades } \\
\hline 1 & $\begin{array}{l}\text { Apresentação do professor/monitor, observador e de cada participante; realização de contrato de sinceridade, } \\
\text { espontaneidade e privacidade do trabalho; definição do objetivo de reabilitaçáo e melhoria da memória e capacidade } \\
\text { funcional, deixando clara a noção de que não se trata de "oficinas de informática", mas que a informática será usada } \\
\text { como ferramenta digital e, por isso, serão aprendidos aspectos básicos do uso do computador e da Internet; }\end{array}$ \\
& Explicação básica com prática no uso do mouse e ferramenta de desenho livre; \\
3 & Uso do mouse, desenho livre, uso de apresentador de fotos; \\
4 & Uso do mouse, desenho livre, uso de apresentador de fotos, início de uso de jogos (quebra cabeça, paciência, campo \\
5 & minado) sem tempo marcado; \\
6 & Continuação dos jogos; \\
7 & Introdução de Jogos com tempo marcado; \\
8 & Jogos com tempo marcado; \\
9 & Aprendizado de browser, navegação; \\
10 & Aprendizado de browser, busca por palavras e textos; \\
11 e 12 & Aprendizado de browser busca por imagens; \\
13 e 14 & Aprendizado completo de browser (palavras e imagens); \\
15 a 19 & Aprendizado completo de browser (palavras e imagens) e treinamento de navegação; \\
20 & Correio eletrônico; \\
\hline
\end{tabular}

vida profissional?; 2- Qual(is) o(s) benefício(s) de ter sido monitor do projeto em sua vida pessoal?

\section{Tratamento dos Dados}

Para a interpretação das informações que foram obtidas pelo diário de campo e pelas entrevistas, foi utilizada a técnica de análise de conteúdo. Esse tipo de análise é realizado em três etapas (Bardin, 2009): (1) Pré-análise - primeiros contatos com os dados, a formulação de objetivos, a definição dos procedimentos a serem seguidos e a preparação formal do material; (2) Exploração do material - cumprimento das decisões anteriormente tomadas e (3) Tratamento dos resultados - lapidação dos dados, tornando-os significativos.

\section{Aspectos Éticos}

Para realização dessa pesquisa, foram cumpridos todos os princípios éticos (resolução 196 do Conselho Nacional de Saúde). O Comitê de Ética em Pesquisas em Seres Humanos da UFSC aprovou a pesquisa (protocolo $\mathrm{n}^{\circ} 866664 / 2015$ ). Todos os idosos e monitores que aceitaram participar das pesquisas assinaram o Termo de Consentimento Livre e Esclarecido.

\section{RESULTADOS E DISCUSSÃO}

Para melhor entendimento, descrição dos resultados e discussão, os mesmos serão apresentados separadamente de acordo com os sujeitos do estudo: (1) Benefícios percebidos por pessoas idosas participantes da Oficina da Lembrança e (2) Benefícios percebidos pelos monitores da Oficina da Lembrança.

\section{Benefícios Percebidos pelos Idosos Participantes da Oficina da Lembrança}

Dos 10 idosos participantes desta pesquisa, a maioria era de mulheres, média de idade de 70,3 anos $( \pm 4,2)$, casados, aposentados, com renda mensal entre 2 a 35 salários mínimos. Todos os idosos possuíam ensino médio completo. Cinco usavam o computador com frequência (todos ou quase todos os dias da semana) antes de participar do projeto e quatro praticavam atividade física regular no lazer. Os objetivos percebidos pelos idosos participantes das Oficinas foram divididos entre os relativos ao uso dos computadores e da prática de atividades físicas realizadas.

Benefícios do Uso de Computadores. O Quadro 2 apresenta os benefícios percebidos pelos idosos em relação à aprendizagem do uso de computadores.

Aprendizagem do Uso de Computadores. Os idosos de hoje nasceram e cresceram em uma sociedade sem muitos recursos tecnológicos, ou seja, viveram a maior parte da vida 
Quadro 2

Beneficios percebidos pelos idosos participantes da Oficina da Lembrança em relação à aprendizagem do uso dos computadores. Florianópolis, Santa Catarina, Brasil, 2014.

\begin{tabular}{|c|c|}
\hline \multirow{3}{*}{ Aprendizagem do uso de computadores } & “...não sabia nada de PC e aprendi. Faz a gente aprender bastante”. [Idoso1] \\
\hline & $\begin{array}{l}\text { "Não tenho muita capacidade, mas agora estou sentindo a sequência da aprendizagem no } \\
\text { computador". [Idoso5] }\end{array}$ \\
\hline & "Aprendi muito a mexer nos computadores e na internet..." [Idoso7] \\
\hline \multirow{3}{*}{ Melhora da memória } & “...melhorou a memória. Sente a memória melhor, forçada.” [Idoso1] \\
\hline & $\begin{array}{l}\text { "Tratamento da mente, treinar, memorizar mais rápido. Gostei de treinar, usar e aperfeiçoar a } \\
\text { memória, aperfeiçoar." [Idoso3] }\end{array}$ \\
\hline & "Movimentação da mente..." [Idoso4] \\
\hline \multirow{3}{*}{ Sociabilização } & $\begin{array}{l}\text { "relembrar coisas que estavam caindo no esquecimento...é muito valoroso colocar a nossa capacidade } \\
\text { em teste, o raciocínio, a memória, o esquecimento. É bom relembrar o passado." [Idoso6] }\end{array}$ \\
\hline & “...as aulas me estimulavam a lembrar de coisas do passado que eu nem pensava mais.” [Idoso8] \\
\hline & "Sinto que minha memória mudou, lembro mais das coisas..." [Idoso10] \\
\hline \multirow[b]{2}{*}{ Ter uma atividade de lazer } & “O computador é descontraído, é um lazer”. [Idoso3] \\
\hline & $\begin{array}{l}\text { "Ter um lazer. Ao invés de ficar em casa pensando em doença, estamos aqui aprendendo e nos } \\
\text { divertindo". [Idoso6] }\end{array}$ \\
\hline
\end{tabular}

sem utilizar esse recurso e, por isso, têm maior resistência e dificuldade com o uso da mesma quando comparadas aos mais jovens, que são introduzidos nesse universo desde o nascimento (Pereira \& Neves, 2011). Além disso, esses autores explicam que as pessoas idosas, por já terem saído do mercado de trabalho e parado de estudar na escola ou universidades, acabam convivendo menos com o uso de computadores.

O município de Florianópolis/SC possui o $3^{\circ}$ maior Índice de Desenvolvimento Humano do Brasil, os principais fatores que contribuem para isso são a longevidade, a renda e a educação de sua população (Brasil, 2010). Apesar desses contribuintes, apenas $20,7 \%$ dos idosos do município sabem utilizar a internet (Medeiros, Xavier \& Schneider, 2012), demonstrando que a exclusão digital ainda é um impasse na vida de muito idosos (Centro de Estudos sobre as Tecnologias da Informação e da Comunicação, 2010).

A aprendizagem do uso de computadores pode auxiliar na redução de limitações físicas, mentais e socioeconômicas associadas ao envelhecimento, aumentando a sociabilização, cidadania, saúde, educação, trabalho, lazer, vida social e estímulo intelectual (Pereira \& Neves, 2011; Ala-Mutka et al., 2008). A Oficina da Lembrança surge como fonte de aprendizagem do uso de computadores para os idosos, pois proporciona convívio com as ferramentas computacionais, oportunizando o aprendizado tecnológico e promovendo a inclusão digital.

Melhora da memória. Estudos mostram que o uso de computadores pode melhorar significativamente a memória de pessoas idosas, além de prevenir transtornos cognitivos leves e demência (Singh-Manoux et al., 2012; Burlá et al., 2013; Norton et al., 2014).

Evidências sugerem que o treinamento cognitivo em idosos auxilia na manutenção ou melhora da performance cognitiva (Borella, Carretti, Zanoni, Zavagnin \& De
Beni, 2013). Estudo realizado com mulheres idosas com Comprometimento Cognitivo Leve, do qual avaliou o efeito do treinamento cognitivo mediante o uso do computador, encontrou melhora no tempo de reação, número de erros e na velocidade de processamento no grupo intervenção (Fatemeh, Arsalan, \& Parvin, 2016).

Tais resultados apontam para uma possível plasticidade cerebral ainda na velhice, sendo que o uso desse tipo de ferramenta é possivelmente uma boa estratégia para prevenir perdas de memória, funcionando, inclusive, como um método complementar de tratamento para déficits cognitivos.

Sociabilização. O contato com acadêmicos de graduação, pós-graduação e com os professores responsáveis pelo projeto também foi visto como fator positivo pelos idosos para a sociabilização, além do contato com outros de sua geração.

O uso de computadores aumenta a vida social de pessoas idosas (Medeiros et al., 2012; Ala-Mutka et al., 2008; d'Orsi, Xavier \&, Ramos, 2011), tende a aumentar a comunicação com familiares e amigos, a construção de novas amizades e fortalece as relações intergeracionais (Centro de Estudos sobre as Tecnologias da Informação e da Comunicação, 2010). O uso de computadores é considerado uma ferramenta importante, capaz de auxiliar na prevenção da exclusão social e da depressão, visto que pessoas idosas que fazem uso do computador sentem-se menos excluídos na sociedade, tendo em vista que a sociedade está cada vez mais tecnológica (Pereira \& Neves, 2011).

Idosos que procuram por programas de inclusão digital estão em busca de processos de inclusão social, seja pelo sentimento de pertencimento à sociedade, que o conhecimento da tecnologia pode influenciar, ou pela maior quantidade e qualidade de contatos que as ferramentas de comunicação suportadas pela internet podem lhes oferecer (Pereira \& Neves, 2011). Sayago \& Blat (2010), ao estudarem 388 
pessoas idosas, evidenciaram que aqueles que faziam uso do computador tiveram maior interação com amigos e/ou familiares e maior integração na sociedade moderna.

Ter uma atividade de lazer. Atualmente, o uso de computadores, principalmente a internet e os jogos eletrônicos, se tornou uma ótima forma de lazer (Medeiros et al., 2012; d'Orsi et al., 2011). No entanto, o lazer digital é pouco explorado pelas pessoas idosas, tendo em vista que a maioria prefere realizar atividades como assistir televisão e caminhar (Ala-Mutka et al., 2008). Por não saberem utilizar o computador, os idosos perdem a oportunidade de conhecer um novo mundo, digital e tecnológico, com infinitas possibilidades de lazer (Pereira \& Neves, 2011).

Benefícios da prática de atividade física. $O$ Quadro 3 apresenta os benefícios percebidos pelos idosos em relação à prática de atividade física.

Emagrecimento. A prática de atividade física pode auxiliar no controle do peso corporal, podendo promover redução de peso, da porcentagem de gordura corporal e da relação cintura/quadril (American College of Sports Medicine, 2009; Miura, 2011), influenciando, assim, o emagrecimento das pessoas (Lageros, 2009).

$\mathrm{O}$ fato de os idosos perceberem o emagrecimento como um benefício do projeto relacionado à prática de exercícios físicos é muito importante, pois, apesar do objetivo principal do mesmo ser manter, estimular e/ou reabilitar a função cognitiva, a obesidade é um dos fatores associados ao declínio cognitivo (Williams, Plassman, Burke, \& Holsinger, 2010), assim, esse efeito pode auxiliar na função cognitiva dos participantes do projeto.

Sensação de bem-estar e melhora da qualidade de vida. Pesquisa de Souza \& Duarte (2013) traz que a qualidade de vida é uma avaliação subjetiva que engloba felicidade, satisfação, estado de espirito, afetividade e bem-estar. Os mesmos autores explicam que o envolvimento social, qualquer que seja, pode promover melhora do bem-estar.

Segundo o American College of Sports Medicine (2009), pessoas que possuem estilo de vida ativo, com prática de atividades físicas regulares, têm melhor percepção de qualidade de vida e do bem-estar subjetivo quando comparadas a pessoas inativas fisicamente. Esse fato se deve aos vários benefícios proporcionados pela prática de atividades físicas, entre eles a menor prevalência de morbidades, de uso de medicamentos, melhora da capacidade física e mental, aumento da autoestima e sociabilização (Lageros, 2009).

Pesquisa experimental com 44 indivíduos (média de idade de 59,8 $\pm 5,78$ anos) mostrou que idosos que realizaram 15 semanas de caminhada (10.000 passos diários) obtiveram melhora significativa no desempenho cardiovascular, autoestima e no bem-estar quando comparados a sujeitos inativos fisicamente (Miura, 2011).

Sociabilização. A prática de atividades físicas em grupo pode proporcionar maior apoio social, relações pessoais e a formação de novas amizades (American College of Sports Medicine, 2009; Lageros, 2009). Além disso, idosos convivem pouco com pessoas mais novas e o fato de eles estarem em constante relação com os monitores do projeto pode elevar o sentimento de sociabilização desses idosos.

A convivência intergeracional favorece o processo de inclusão social e ressocialização de pessoas mais velhas, contribuindo no combate da discriminação relacionada à idade. Dessa forma, os programas intergeracionais auxiliam na construção da coesão social, permitindo que os participantes mais velhos utilizem todo o seu potencial e que os mais novos incorporem em suas vidas maior conhecimento, valores e cultura (Butts, 2007).

Melhora da aptidão física. O aumento da idade pode estar associado ao declínio da aptidão física, pois o processo de envelhecimento ocasiona a diminuição da capacidade aeróbica, força muscular, flexibilidade, equilíbrio, tempo de reação de movimento, agilidade e coordenação motora (American College of Sports Medicine, 2009; Lageros, 2009). Embora a prática de atividades físicas não possa interromper o processo biológico do envelhecimento, essa pode minimizar os efeitos biopsicossociais advindos do

Quadro 3.

Beneficios percebidos pelos idosos participantes da Oficina da Lembrança em relação à prática de atividade fisica. Florianópolis, Santa Catarina, Brasil, 2014.

\begin{tabular}{|c|c|}
\hline \multirow{2}{*}{ Emagrecimento } & "Exercícios foram bons, emagreci $2 \mathrm{~kg} "$. [Idoso1] \\
\hline & "Estou me achando mais magro..." [Idoso7] \\
\hline \multirow{3}{*}{$\begin{array}{l}\text { Sensação de bem estar e melhora } \\
\text { da qualidade de vida }\end{array}$} & “Atividade física passa energia, faz bem”. [Idoso2] \\
\hline & "Depois do exercício me sinto bem, fico mais leve, mais agradável". [Idoso5] \\
\hline & "Os exercícios proporcionam melhor qualidade de vida..." [Idoso9] \\
\hline \multirow[b]{2}{*}{ Sociabilização } & “...interação com os outros, principalmente as brincadeiras”. [Idoso9] \\
\hline & $\begin{array}{l}\text { "Gosto de fazer os exercícios porque nos aproximam dos colegas e dos professores..." } \\
\text { [Idoso10] }\end{array}$ \\
\hline Melhora da aptidão física & $\begin{array}{l}\text { "Os exercícios tiram a areia do pescoço. O velhinho não tá conseguindo se espichar } \\
\text { todo". [Idoso4] }\end{array}$ \\
\hline \multirow[t]{2}{*}{ Melhora da saúde } & $\begin{array}{l}\text { "Estou fazendo alguma coisa por mim, pra levar a vida mais suave e com mais } \\
\text { saúde". [Idoso2] }\end{array}$ \\
\hline & "Estou sempre procurando ficar mais jovem e saudável fazendo exercícios". [Idoso5] \\
\hline
\end{tabular}


aumento da idade, comuns nessa fase da vida (American College of Sports Medicine, 2009).

A prática de atividade física, mesmo que iniciada em idade avançada e independentemente do seu tempo semanal de prática, está associada a melhores níveis de saúde, de qualidade de vida e de variáveis antropométricas, reduz o número de medicamentos prescritos e melhora os níveis de aptidão física (Lageros, 2009).

Nesse sentido, a atividade física pode melhorar a aptidão física, promovendo a saúde das pessoas idosas (Miura, 2011). Van Roie et al. (2010), ao pesquisarem 180 idosos sedentários, dividindo-os em grupo controle (não fazem exercícios), grupo de exercícios supervisionados e grupo de exercícios não supervisionados mostraram que realizar exercícios supervisionados melhora a aptidão física de pessoas idosas.

Melhora da saúde. A prática regular de atividades físicas é apontada como um dos fatores comportamentais que mais contribui para um envelhecimento com saúde, assim, este comportamento vem sendo amplamente recomendado por profissionais e organizações de saúde como uma forma de promover a saúde de pessoas idosas e minimizar os efeitos biopsicossociais advindos do aumento da idade (American College of Sports Medicine, 2009; Lageros, 2009; Miura, 2011).

\section{Benefícios Percebidos pelos Monitores da Oficina da Lembrança}

Os benefícios percebidos pelos monitores do programa para sua formação profissional estão descritos no Quadro 4. Os monitores tinham entre 20 e 24 anos de idade, sendo quatro do sexo masculino e dois do feminino.

Contato com idosos. Projetos de extensão que envolvem processos intergeracionais constituem excelente oportunidade para os alunos conhecerem ainda mais sobre uma faixa etária que está aumentando progressivamente na nossa população, fomentando o compromisso dos alunos com pessoas de maior idade. Além do mais, a formação da personalidade dos alunos como futuros profissionais requer o contato com os diferentes grupos inseridos na sociedade, para que o processo de conhecimento ocorra de maneira efetiva (Butts, 2007). Dessa forma, é necessário que esses futuros profissionais tenham compreensão dos problemas sociais sobre os quais terão que atuar e de seus impactos sociais, sendo que tal entendimento pode ser adquirido mediante as atividades de extensão universitária, que proporcionam o contato direto com as grandes questões contemporâneas (Fórum de Pró-reitores de Extensão das Universidades Públicas Brasileiras, 2012).

Melhora da relação com as pessoas/pacientes. Entre os conhecimentos adquiridos durante a universidade, incluem-se habilidades de comunicação, em que o aluno aprende a comunicar-se, especialmente com os pacientes. Para uma boa relação médico-paciente, alguns aspectos importantes são necessários, como empatia, saber lidar com estresse e ansiedade e paciência (Shapiro, 2008). Uma boa comunicação médico-paciente está associada a melhor adesão aos planos de tratamento, maior compreensão por parte dos pacientes, e maior satisfação tanto dos médicos quanto dos pacientes (Fórum de Pró-reitores de Extensão das Universidades Públicas Brasileiras, 2012).

Preparação para situações diversas na vida profissional e pessoal. Os monitores disseram que a participação no projeto ajudou a prepará-los para diversas situações que podem acontecer na vida profissional e pessoal. Por ser um programa de extensão universitária, onde os alunos ministram aulas e propõem atividades a pessoas idosas, a Oficina da Lembrança pode funcionar como ambiente de interação, aprendizado, troca de experiências

Quadro 4.

Beneficios percebidos pelos monitores da Oficina da Lembrança. Florianópolis, Santa Catarina, Brasil, 2014.

\begin{tabular}{|c|c|}
\hline Contato com idosos & $\begin{array}{l}\text { "Contato com faixa etária que cada vez está crescendo mais e que terei bastante contato no } \\
\text { futuro como profissional, esse contato da oficina ajuda a me preparar". [Monitor1] }\end{array}$ \\
\hline \multirow{3}{*}{$\begin{array}{l}\text { Melhora da relação com } \\
\text { as pessoas/pacientes }\end{array}$} & $\begin{array}{l}\text { "...melhora a relação com as pessoas, o que é muito necessário na faculdade. Aprendi a ter } \\
\text { paciência e a lidar melhor com as pessoas". [Monitor2] }\end{array}$ \\
\hline & $\begin{array}{l}\text { "Melhorou a relação com os pacientes, como abordar e até mesmo como conversar com as } \\
\text { pessoas de uma maneira geral". [Monitor3] }\end{array}$ \\
\hline & $\begin{array}{l}\text { "Melhor interação com as pessoas...melhora habilidades de comunicação...mais paciência". } \\
\text { [Monitor4] }\end{array}$ \\
\hline \multirow{2}{*}{$\begin{array}{l}\text { Preparação para situações diversas } \\
\text { na vida profissional e pessoal }\end{array}$} & $\begin{array}{l}\text { "Ficar mais preparado para situações diversas, ter jogo de cintura. Capacidade de resolução } \\
\text { de problemas, manejar problemas de forma mais tranquila..." [Monitor3] }\end{array}$ \\
\hline & $\begin{array}{l}\text { "....aprendi a ter menos pressa em relação ao tempo, a saber esperar, ter mais paciência e lidar } \\
\text { com os problemas do dia a dia". [Monitor5] }\end{array}$ \\
\hline \multirow[b]{2}{*}{ Ter mais conhecimentos sobre idosos } & $\begin{array}{l}\text { “...estimula a querer pesquisar sobre os assuntos e pensar no próprio futuro como idoso". } \\
\text { [Monitor1] }\end{array}$ \\
\hline & $\begin{array}{l}\text { "Pude perceber certas dificuldades motoras e cognitivas dos idosos e isso me trouxe mais } \\
\text { conhecimentos, como orientar certos cuidados, enxergar melhor essas dificuldades. Pude } \\
\text { aprender um pouco sobre os idosos..." [Monitor5] }\end{array}$ \\
\hline
\end{tabular}


e proporcionar benefícios aos acadêmicos tanto na vida profissional quanto na pessoal (Fórum de Pró-reitores de Extensão das Universidades Públicas Brasileiras, 2012). Sabe-se que estudantes da área médica necessitam lidar com questões emocionais complexas e que, muitas vezes, não são ensinados de maneira correta para isso (Shapiro, 2008). A participação de estudantes de medicina em projetos de extensão como esse pode promover o desenvolvimento e aprimoramento de habilidades exigidas na vida acadêmica e profissional, preparando-os melhor para situações futuras diversas (Fórum de Pró-reitores de Extensão das Universidades Públicas Brasileiras, 2012).
Ter mais conhecimentos sobre idosos. Nas Oficinas, os jovens passam a ser tutores de pessoas mais velhas em atividades que necessitas de tecnologia, por exemplo, em que transmitem conhecimentos adquiridos rotineiros ao seu dia a dia, promovendo relações intergeracionais. Essa intergeracionalidade enriquece a troca de experiências e desperta a curiosidade para o conhecimento do novo (Butts, 2007; França, Silva \&, Barreto, 2010). No caso dos monitores, o contato estimulou-os a querer conhecer e estudar mais sobre idosos, além de auxiliá-los a enxergar as dificuldades vivenciadas por essa população, suas capacidades e papel social.

\section{CONSIDERAÇÕES FINAIS}

Os idosos perceberam vários benefícios após a participação na Oficina da Lembrança, tais como: aprendizagem do uso de computadores, melhora da memória, sociabilização, ter uma atividade de lazer, emagrecimento, melhora da qualidade de vida, bem-estar e saúde. Já os monitores perceberam os seguintes benefícios na sua formação profissional: contato com idosos, melhora da relação com pessoas/pacientes, preparação para situações diversas na vida pessoal e profissional e ter mais conhecimentos sobre idosos.

Assim, os resultados deste estudo mostram que programas de manutenção, estimulação e/ou reabilitação cognitiva podem proporcionar benefícios à população idosa. Além disso, este estudo mostra que projetos de extensão podem auxiliar na formação e na atuação futura de acadêmicos de Medicina.

$\mathrm{O}$ ambiente das Oficinas proporcionou um convívio amigável e acolhedor, que propiciou a sociabilização e a aprendizagem, além da troca intergeracional (idosos e estudantes), que beneficia ambos os lados, mas especialmente os idosos. Mesmo com suas limitações funcionais e cognitivas os idosos mantiveram-se sempre dispostos a participar, aprender e ensinar.

Entre os pontos positivos do estudo, podemos citar seu delineamento, que selecionou de forma aleatória os idosos provenientes de um amplo inquérito populacional para participar do projeto, a intergeracionalidade promovida entre os participantes e os alunos monitores, a utilização de ferramentas digitais e a importância social que projetos universitários como este possuem. Como limitações, destacam-se o número pequeno de participantes, o curto tempo de acompanhamento e a não exclusão de idosos que eram previamente ativos fisicamente e já faziam uso de computadores, o que pode ter interferido nos relatos dos mesmos.

Sugere-se que novos estudos sejam realizados, incluindo um número maior de participantes e associando análise do desempenho cognitivo de cada idoso com os benefícios percebidos pelos mesmos, avaliando se o declínio cognitivo pode interferir na percepção dos benefícios do programa.

\section{REFERÊNCIAS}

Ala-Mutka, K., Malanowski, N., \& Punie, Y. (2008). Active ageing and the potential of ICT for learning. Sevilha/Espanha: JCREuropean Commission.

American College of Sports Medicine. (2009). Exercise and physical activity for older adults. Medicine Science of Sports and Exercise, 41(7), 1510-1530.

Bardin, L. (2009). Análise de Conteúdo. Lisboa: Ed. Porto LDA.

Barnes, D. E., Santos-Modesitt, W., Poelke, G., Kramer, A. F., Castro, C., Middleton, L. E., \& Yaffe, K. (2013). The Mental Activity and exercise (MAX) Trial. Journal of the American Medical Association International Medicine, 173(9), 797-804.

Blondell, S. J., Hammersley-Mather, R., \& Veerman, J. L. (2014). Does physical activity prevent cognitive decline and dementia?: A systematic review and meta-analysis of longitudinal studies. BMC Public Health, 14, 510.

Borella, E., Carretti, B., Zanoni, G., Zavagnin, M., \& De Beni, R. (2013). Working memory training in old age: an examination of transfer and maintenance effects. Archives of Clinical Neuropsychology, 28(4), 331-47.
Brasil. Instituto de Pesquisa Econômica Aplicada. (2010). Programa das Nações Unidas para o Desenvolvimento no Brasil. Brasília: Fundação João Pinheiro.

Burlá, C., Camarano, A. A., Kanso, S., Fernandes, D., \& Nunes, R. (2013). Panorama prospectivo das demências no Brasil: um enfoque demográfico. Ciência e Saúde Coletiva, 18(10), 2949-2956.

Butts, D. M. (2007). Programas intergeneracionales e inclusión social de las personas mayores. In M. Sánchez (Org.), Programas Intergeracionales: Hacia una sociedad para todas las edades. Barcelona: Fundación "La Caixa".

Centro de Estudos sobre as Tecnologias da Informação e da Comunicação. (2010). Pesquisa sobre o uso das tecnologias de informação e comunicação no Brasil: TIC Domicílios e TIC Empresas. São Paulo: Comitê Gestor da Internet no Brasil.

Cotelli, M., Manenti, R., Zanetti, O., \& Miniussi, C. (2012). Nonpharmacological intervention for memory decline. Frontiers in Human Neuroscience, 6, 46. 
Diamond, A., \& Ling, D. S. (2016) Conclusions about interventions, programs, and approaches for improving executive functions that appear justified and those that, despite much hype, do not. Developmental Cognitive Neuroscience, 18(4), 34-48.

d'Orsi, E., Xavier, A. J., \& Ramos, L. R. (2011). Work, social support and leisure protect the elderly from functional loss: EPIDOSO study. Revista de Saúde Pública, 45(4), 685-692.

Fatemeh, H., Arsalan, D., \& Parvin, B. (2016). Effect of brain training on cognitive performance in elderly women diagnosed with mild cognitive impairment. Caspian Journal of Neurological Sciences, 2(7), 25-31.

Fonseca, J. A. S., Ducksbury, R., Rodda, J., Whitfield, T., Nagaraj, C., Suresh, K ... Walker, Z. (2015). Factors that predict cognitive decline in patients with subjective cognitive impairment. International Psychogeriatrics, 27(10), 1671-1677.

Fórum de Pró-reitores de Extensão das Universidades Públicas Brasileiras. (2012). Política Nacional de Extensão Universitária. Manaus: Fórum de Pró-reitores de Extensão das Universidades Públicas Brasileiras.

França, L. H. F. P., Silva, A. M. T. B., \& Barreto, M. S. L. (2010). Programas intergeracionais: quão relevantes eles podem ser para a sociedade brasileira? Revista Brasileira de Geriatria e Gerontologia, 13(3): 519-531.

Klusmann, V., Evers, A., Schwarzer, R., Schlattmann, P., Reischies, F. M., Heuser, I., \& Dimeo, F. C. (2010). Complex mental and physical activity in older women and cognitive performance: A 6-month randomized controlled trial. The Journal of Gerontology: A Biological Sciences and Medical Sciences, 65(6), 680-8.

Krug, R. R., Ono, L. M., Quialheiro, A., d'Orsi, E., Ramos, L. R., \& Xavier, A. J. (2015). A Stimulation and rehabilitation program: Oficina da Lembrança. Revista Brasileira de Atividade Física \& Saúde, 20(5), 534-540.

Lageros, Y. T. (2009). Physical activity. The more we measure, the more we know to measure. European Journal Epidemiology, 24, 119-122.

Lautenschlager, N. T., Cox, K. L. (2013). Can participation in mental and physical activity protect cognition in old age?: Comment on "The Mental Activity and eXercise (MAX) trial: a randomized controlled trial to enhance cognitive function in older adults". JAMA International Medicine, 173(9), 805-6.

Livingston, G., Sommerlad, A., Orgeta, V., Costafreda, S. G., Huntley, J., Ames, D., ... Mukadam, N. (2017). Dementia prevention, intervention, and care. Lancet, 390, 2673-734.

Medeiros, F., Xavier, A., \& Schneider I. (2012). Digital inclusion and functional capacity of older adults living in Florianópolis, Santa Catarina, Brazil (EpiFloripa 2009-2010). Revista Brasileira de Epidemiologia, 15(1), 106-122.

Miura, K. (2011). Epidemiology and prevention of hypertension in Japanese: How could Japan get longevity? European Association for Predictive, Preventive and Personalised Medicina, 2(1), 59-64.

Ngandu, T., Lehtisalo, J., Solomon, A., Levälahti, E., Ahtiluoto, S., Antikainen, R., ... Kivipelto, M. (2015). A 2 year multidomain intervention of diet, exercise, cognitive training, and vascular risk monitoring versus control to prevent cognitive decline in at-risk elderly people (FINGER): A randomised controlled trial. Lancet, 6736, 1-9.

Norton, S., Matthews, F. E., Barnes, D. E., Yaffe, K., \& Brayne, C. (2014). Potential for primary prevention of Alzheimer's disease: An analysis of population-based data. Lancet Neurologic, 13, 788-94.

Pereira, C., \& Neves, R. (2011). Os idosos na aquisição de competências TIC. Educação, Formação \& Tecnologias, 4(2), 15-24.

Sayago, S., \& Blat, J. (2010). Telling the story of older people e-mailing: An ethnographical study. International Journal of Human-Computer Study, 68(1-2), 105-120.

Shapiro, J. (2008). Walking a mile in their patients' shoes: Empathy and othering in medical students' education. Philos Ethics Humanit Medicine, 12(3), 10-19.

Singh-Manoux, A., Kivimaki, M., Glymour, M. M., Elbaz, A., Berr, C., Ebmeier, K. P., ... Dugravot, A. (2012). Timing of onset of cognitive decline: Results from Whitehall II prospective cohort study. BMJ, 344(04), d7622.

Souza, L. K., \& Duarte, M. G. (2013). Amizade e bem-estar subjetivo. Psicologia: Teoria e Pesquisa, 29(4), 429-436.

Tardif, S., \& Simard, M. (2011). Cognitive stimulation programs in healthy elderly: A review. Interntional Journal of Alzheimer's Disease, 1, 1-13.

Van Roie, V., Delecluse, C., Opdenacker, J., De Bock, K., Kennis, E., \& Boen, F. (2010). Effectiveness of a lifestyle physical activity versus a structured exercise intervention in older adults. Journal Aging and Physical Activity, 18, 335-352.

Wang, H-X., MacDonald, S. W. S., Dekhtyar, S., \& Fratiglioni, L. (2017) Association of lifelong exposure to cognitive reserveenhancing factors with dementia risk: A community-based cohort study. PLoS Medicine, 14(3), e1002251

Williams, J., Plassman, B., Burke, J., \& Holsinger, T. (2010). Preventing Alzheimer's disease and cognitive decline. Health, 193(10-E005), 3-727

Woods, B., Aguirre, E., Spector, A. E., \& Orrell, M. (2012). Cognitive stimulation to improve cognitive functioning in people with dementia (Review). The Cochrane Database of Systematic Reviews, 2(15).

World Health Organization. (2012). Dementia: A public health priority. Geneva: WHO.

Xavier, A. J. (2002). Cognição, Interação e Envelhecimento: Estudo Exploratório a partir de Oficinas de Internet (Dissertação de Mestrado). Programa de Pós-Graduação em Ciências da Computação, Universidade Federal de Santa Catarina, Florianópolis, SC, Brasil.

Xavier, A. J., d'Orsi, E., Oliveira, C. M., Orrell, M., Demakakos, P., Biddulph, J. P., \& Marmot, M. G. (2014). English Longitudinal Study of Aging: Can internet/e-mail use reduce cognitive decline? The Journal of Gerontology: A Biological Sciences and Medical Sciences, 12(69), 1117-21.

Zanello, V., Silva, L. C., \& Henderson, G. (2015). Saúde mental, gênero e velhice na instituição geriátrica. Psicologia: Teoria e Pesquisa, 31(4), 534-550. 